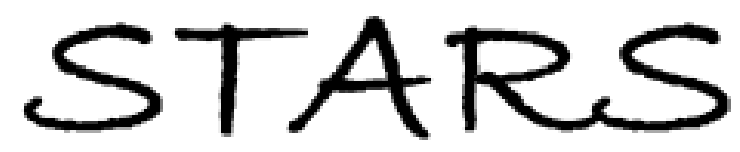

University of Central Florida

STARS

$1-1-2006$

\title{
A multiwavelength radial velocity search for planets around the brown dwarf LP 944-20
}

\author{
E. L. Martin \\ University of Central Florida \\ E. Guenther \\ M. R. Zapatero Osorio \\ H. Bouy \\ R. Wainscoat
}

Find similar works at: https://stars.library.ucf.edu/facultybib2000 University of Central Florida Libraries http://library.ucf.edu

This Article is brought to you for free and open access by the Faculty Bibliography at STARS. It has been accepted for inclusion in Faculty Bibliography 2000s by an authorized administrator of STARS. For more information, please contactSTARS@ucf.edu.

\section{Recommended Citation}

Martin, E. L.; Guenther, E.; Zapatero Osorio, M. R.; Bouy, H.; and Wainscoat, R., "A multiwavelength radial velocity search for planets around the brown dwarf LP 944-20" (2006). Faculty Bibliography 2000s. 6406. https://stars.library.ucf.edu/facultybib2000/6406

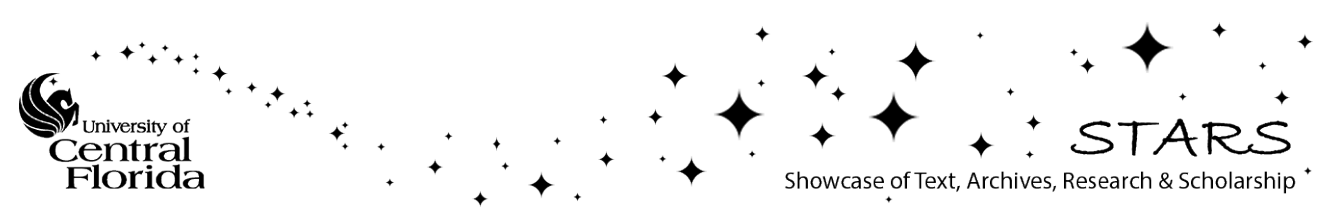




\title{
A MULTIWAVELENGTH RADIAL VELOCITY SEARCH FOR PLANETS AROUND THE BROWN DWARF LP 944-20
}

\author{
E. L. Martín, ${ }^{1,2}$ E. Guenther,${ }^{3}$ M. R. Zapatero Osorio, ${ }^{4}$ H. Bouy, ${ }^{1}$ and R. Wainscoat \\ Received 2006 April 10; accepted 2006 April 25; published 2006 May 26
}

\begin{abstract}
The nearby brown dwarf LP 944-20 has been monitored for radial velocity variability at optical and nearinfrared wavelengths using the VLT/UVES and the Keck/NIRSPEC, respectively. The UVES radial velocity data obtained over 14 nights spanning a baseline of 841 days show significant variability with an amplitude of 3.5 $\mathrm{km} \mathrm{s}^{-1}$. The periodogram analysis of the UVES data indicates a possible period between 2.5 and $3.7 \mathrm{hr}$, which is likely due to the rotation of the brown dwarf. However, the NIRSPEC data obtained over 6 nights show an rms dispersion of only $0.36 \mathrm{~km} \mathrm{~s}^{-1}$ and do not follow the periodic trend. These results indicate that the variability seen with UVES is likely to be due to rotationally modulated inhomogeneous surface features. We suggest that future planet searches around very low mass stars and brown dwarfs using radial velocities will be better conducted in the near-infrared than in the optical.
\end{abstract}

Subject heading: stars: low-mass, brown dwarfs

\section{INTRODUCTION}

Brown dwarfs (BDs) are objects with very low masses $\left(M<0.075 M_{\odot}\right)$ that develop degenerate cores before being able to settle on the stellar main sequence (Kumar 1963). Large numbers of BDs have been identified in the field, in open clusters and in star-forming regions. These substellar-mass objects could be as numerous as the known stars (Béjar et al. 2001; Chabrier 2003).

The detection of strong emission lines in young BDs, as well as near-infrared, mid-infrared, and millimeter emission exceeding those expected for normal photospheres, strongly indicates that young BDs have disks where planets may form (Barrado y Navascués \& Martín 2003; Klein et al. 2003; Luhman et al. 2005). Moreover, mid-infrared spectroscopic observations obtained with the Spitzer Infrared Spectrograph indicate dust evolution in the disks around young BDs (Apai et al. 2005).

One of the first obvious targets to search for planets around BDs is LP 944-20 [BRI B0337-3535; LEHPM 3451; 2MASS $\left.\mathrm{J} 03393521-3525440 ; J_{2 \mathrm{MASS}}=10.72,(J-K)_{2 \mathrm{MASS}}=1.18\right]$ because it is nearby $(d=5.0 \mathrm{pc}$; Tinney 1998) and young (age 320 Myr; Ribas 2003). This BD was first spotted over 30 years ago (Luyten \& Kowal 1975) as a dim high proper motion red star, and its substellar nature was revealed through the spectroscopic detection of lithium, a trademark of BDs (Magazzù et al. 1993; Tinney 1998). LP 944-20 has a relatively early spectral class for a BD (dM9; Martín et al. 1999), because of its young age, and has a low level of chromospheric and X-ray coronal activity (Martín \& Bouy 2002), although occasional flares have been reported (Rutledge et al. 2000). Nevertheless, LP 944-20 was detected as a radio source, suggesting the presence of strong magnetic activity (Berger et al. 2001).

In this Letter we present a search for giant planets around LP 944-20. We have monitored the radial velocity (RV) of this BD over 20 nights at optical and near-infrared wavelengths. We find that the RV data obtained in the optical are variable, with an amplitude of $3.5 \mathrm{~km} \mathrm{~s}^{-1}$ and a period between 2.5 and

\footnotetext{
${ }^{1}$ Instituto de Astrofísica de Canarias, La Laguna, Tenerife 38200, Spain.

${ }^{2}$ University of Central Florida, Department of Physics, P.O. Box 162385, Orlando, FL 32816.

3 Thuringer Landessternwarte Tautenburg, 07778 Tautenburg, Germany.

${ }^{4}$ LAEFF-INTA, P.O. Box 50727, E-28080, Madrid, Spain.

${ }^{5}$ Institute of Astronomy, University of Hawaii, 2680 Woodlawn Drive, Honolulu, HI 96822.
}

$3.7 \mathrm{hr}$. However, the RV data measured in our near-infrared spectra have an rms dispersion of $0.36 \mathrm{~km} \mathrm{~s}^{-1}$ and do not follow the periodic pattern seen in the optical. We discuss the implications of these results for RV searches for planets around brown dwarfs.

\section{OBSERVATIONS AND RESULTS}

The visible spectroscopic observations of LP 944-20 were obtained with the UV-Visual Echelle Spectrograph (UVES) on the VLT-UT2 (Kueyen) in service mode. The instrumental configuration was the same as described in Guenther \& Wuchterl (2003). This setting covers simultaneously the regions from 667.0 through $854.5 \mathrm{~nm}$ and from 864.0 to $1040.0 \mathrm{~nm}$. It includes the telluric bands between 686.0 and $693.0 \mathrm{~nm}$ and between 760.0 and $770.0 \mathrm{~nm}$. The telluric lines were used as a secondary wavelength reference because they are known to be stable within $15 \mathrm{~m} \mathrm{~s}^{-1}$ (Balthasar et al. 1982; Smith 1982; Caccin et al. 1985). Six UVES RV measurements of LP 94420 have been reported in the literature, and a possible RV variability of this object has been noted (Guenther \& Wuchterl 2003). In this Letter we report nine additional UVES RV points. All of them were computed in the same way as described in detail in previous work (Guenther \& Wuchterl 2003).

Our infrared spectroscopic observations of LP 944-20 were obtained with NIRSPEC on the Keck II telescope. They have already been described in the context of the measurement of rotational broadening (Zapatero Osorio et al. 2006). We obtained one more NIRSPEC RV data point in 2006 January that was not included in the rotational velocity paper. The data reduction was identical to that of the previous spectra. Near-infrared measurements of the heliocentric RV of LP 944-20 were computed via cross-correlation of the NIRSPEC data with spectra of the field vB 10 (GJ 752B, dM8), which was observed with a very similar instrumental configuration in 2001 June 15 and November 2 (see Fig. 1). From our NIRSPEC data, we measured the radial velocity of $\mathrm{vB} 10$ to be $+34.7 \pm 1 \mathrm{~km} \mathrm{~s}^{-1}$, in very good agreement with the various values $\left(+35.4,+35.2,+35.0 \mathrm{~km} \mathrm{~s}^{-1}\right)$ found in the literature (Martín et al. 1996; Mohanty \& Basri 2003). We adopted $+35.0 \mathrm{~km} \mathrm{~s}^{-1}$ for the correlation procedure. In addition, vB 10 is known to be one of the slowest rotators among ultracool dwarfs ( $v \sin i=6.5 \mathrm{~km} \mathrm{~s}^{-1}$; Mohanty \& Basri 2003).

We calculated the cross-correlation function in multiple echelle orders of the NIRSPEC spectra using the task FXCOR 

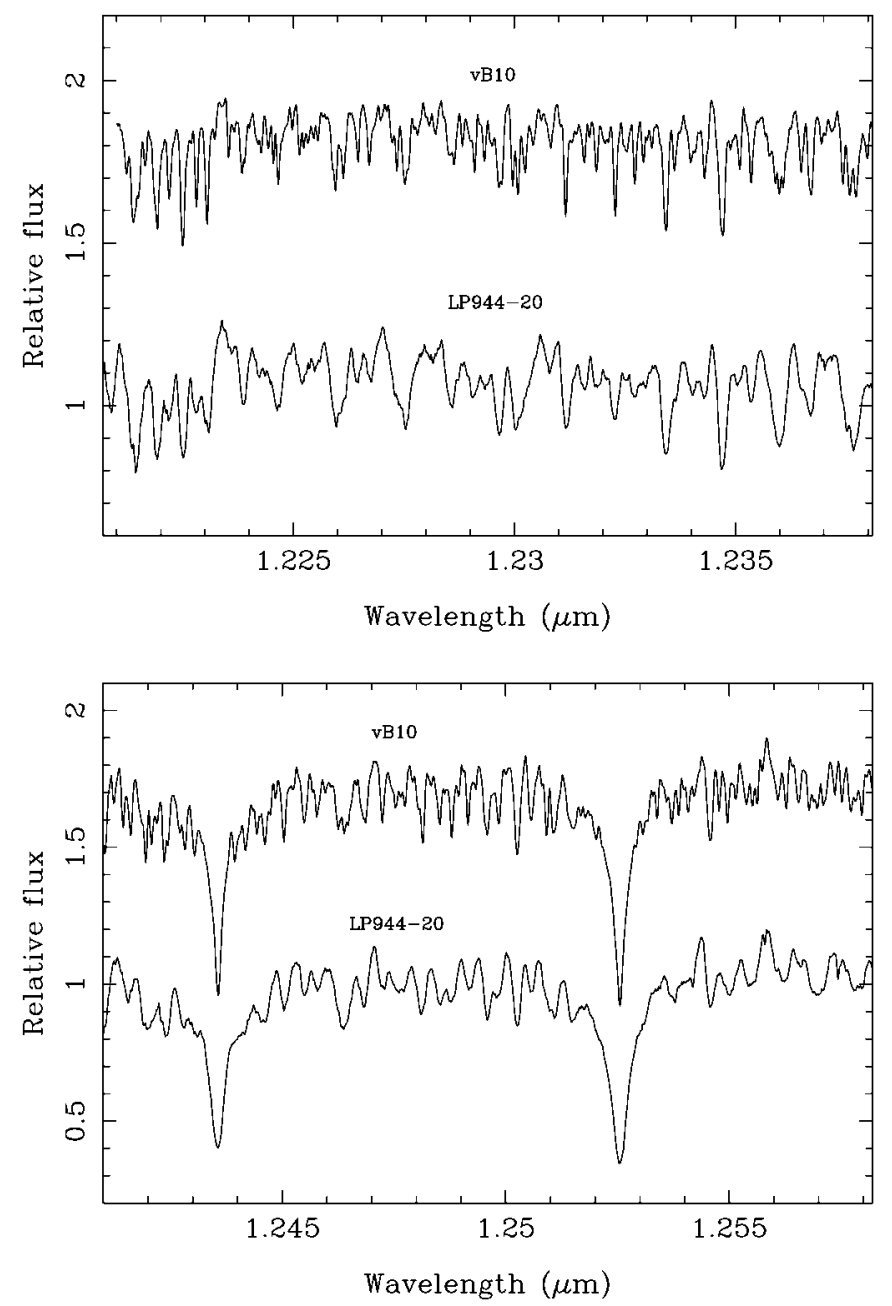

FIG. 1.-Average NIRSPEC spectra for our target (LP 944-20) and the RV template (vB 10). The spectra have been shifted to vacuum wavelength and are offset in the vertical axis. The upper panel shows a NIRSPEC order dominated by $\mathrm{FeH}$ molecular lines, whereas the lower panel shows an order the is dominated by a $\mathrm{K}$ I doublet and $\mathrm{FeH}$ molecular lines. Both of them were used to measure the radial velocity of this brown dwarf.

within IRAF, and we measured velocities by fitting a Gaussian to the cross-correlation peak. We used 4 spectral orders that are free from strong telluric line contamination. Two examples of those orders are shown in Figure 1. These orders include features due to $\mathrm{K} \mathrm{I}, \mathrm{TiO}, \mathrm{FeH}$, and water vapor. We note that given the good signal-to-noise ratio of the spectra and the significant similarity in spectral type between LP 944-20 and vB 10 , the peak of the cross-correlation function is unambiguously identified. The NIRSPEC radial velocities of LP 944-20 shown in Table 1 are the mean of the results in the different orders. The associated error bars correspond to the standard deviation of the mean.

\section{DISCUSSION}

The UVES RV data set shows a significant scatter, and we performed a periodogram analysis of it. The periodogram analysis using the SCARGLE and CLEAN programs (Scargle 1982; Roberts et al. 1987) found a most likely periodicity in the range 2.5-3.7 hr, which is close to the expected rotational period of LP 944-20 $\left(v \sin i=30 \mathrm{~km} \mathrm{~s}^{-1}, P=4.4 \mathrm{hr} \sin i\right.$ for $R=$ $R_{\mathrm{J}}$ ). The CLEAN periodogram is shown in Figure 2. We put the UVES RV data in phase with a period of $3.7 \mathrm{hr}$ (Fig. 3)
TABLE 1

RV DATA FOR LP 944-20

\begin{tabular}{lrcc}
\hline \hline HJD $-2,400,000.0$ & $\begin{array}{c}\text { RV } \\
\left(\mathrm{km} \mathrm{s}^{-1}\right)\end{array}$ & $\begin{array}{c}\sigma \\
\left(\mathrm{km} \mathrm{s}^{-1}\right)\end{array}$ & Instrument \\
\hline $51890.80439 \ldots \ldots$. & 9.04 & 1.00 & NIRSPEC \\
$52189.74347 \ldots \ldots$. & 9.09 & 0.78 & UVES \\
$52213.62965 \ldots \ldots$. & 9.18 & 0.89 & UVES \\
$52214.60754 \ldots \ldots$. & 11.42 & 0.73 & UVES \\
$52224.77031 \ldots \ldots$. & 8.96 & 0.46 & UVES \\
$52236.73029 \ldots \ldots$. & 12.86 & 0.43 & UVES \\
$52237.83445 \ldots \ldots$. & 8.33 & 0.57 & UVES \\
$52915.87534 \ldots \ldots$. & 10.58 & 1.68 & UVES \\
$52920.63108 \ldots \ldots$. & 10.51 & 0.72 & UVES \\
$52927.80874 \ldots \ldots$. & 11.61 & 1.23 & UVES \\
$53006.74733 \ldots \ldots$. & 7.35 & 0.94 & UVES \\
$53010.58583 \ldots \ldots$. & 7.89 & 0.76 & UVES \\
$53010.70228 \ldots \ldots$. & 5.05 & 2.17 & UVES \\
$53017.64839 \ldots \ldots$. & 9.00 & 1.21 & UVES \\
$53022.58255 \ldots \ldots$. & 9.37 & 0.60 & UVES \\
$53030.63830 \ldots \ldots$. & 8.16 & 0.80 & UVES \\
$53669.93870 \ldots \ldots$. & 8.25 & 0.43 & NIRSPEC \\
$53670.97038 \ldots \ldots$. & 8.69 & 0.40 & NIRSPEC \\
$53671.96188 \ldots \ldots$. & 8.61 & 0.48 & NIRSPEC \\
$53672.94703 \ldots \ldots$. & 9.50 & 1.56 & NIRSPEC \\
$53753.70181 \ldots \ldots$. & 8.70 & 0.77 & NIRSPEC \\
\hline
\end{tabular}

and found a good fit with a sinusoid curve with an amplitude of $3.5 \mathrm{~km} \mathrm{~s}^{-1}$.

The NIRSPEC RV data, however, do not follow the periodic trend of the UVES data (Fig. 3), and hence the hypothesis of a planet to explain the UVES RV variations can be discarded because the RV signature of a planet should not depend on wavelength. In fact, our NIRSPEC data have an rms dispersion of $0.36 \mathrm{~km} \mathrm{~s}^{-1}$, which is consistent with observational uncertainties. Using a mass of $60 M_{\mathrm{J}}$ for LP 944-20, we can rule out the presence of any planet with a mass larger than $m \sin i \sim$ $1.0 M_{\mathrm{J}}$ and a period of less than 30 days using the NIRSPEC RV data set.

We confirm that the RV of LP 944-20 is variable at optical

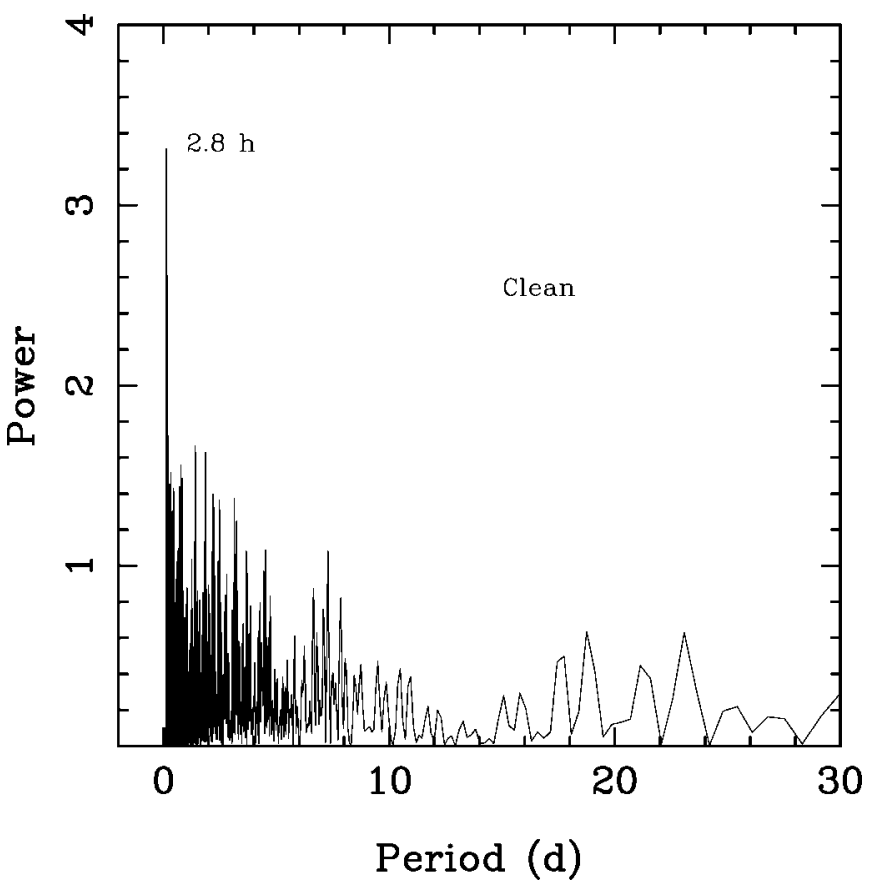

FIG. 2.-CLEAN periodogram analysis of our UVES RV data set of LP 944-20. Five iterations were used, and the gain was 0.1 . The highest peak is marked. 


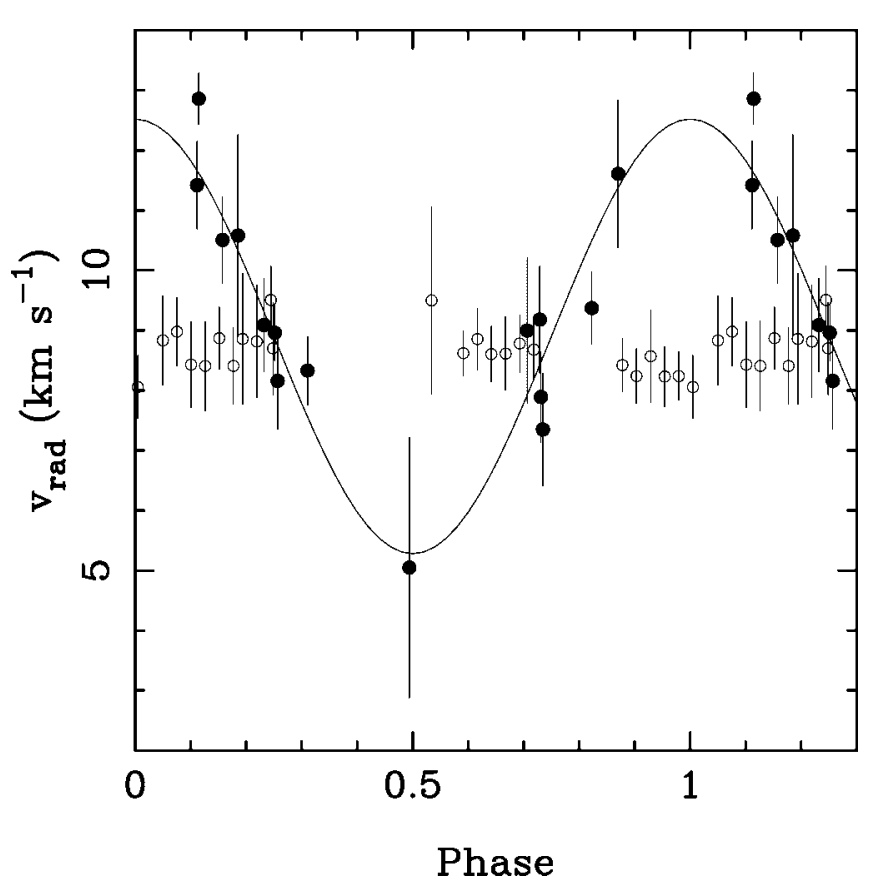

FIG. 3.-RV curve of LP 944-20 folded with a period of $3.7 \mathrm{hr}$ found in the VLT data. A sinusoidal curve provides a good fit for the UVES data (filled circles) but not for the NIRSPEC data (open circles). The individual NIRSPEC points for each spectrum are shown, whereas nightly averages are given in Table 1.

wavelengths as previously reported from UVES observations (Guenther \& Wuchterl 2003). These authors also noted that the $\mathrm{RV}$ variability could not be due to starspots because the filling factor would have to be too large. Now that we have ruled out the existence of a giant planet, there seems to be only one possibility left, which is the presence of inhomogeneous surface features that are not like starspots. Theoretical models have predicted that there could be dust clouds in BDs, and several searches for photometric variability have been performed among late $\mathrm{M}$ and $\mathrm{L}$ dwarfs (Bailer-Jones \& Mundt 2001; Martín et al. 2001; Gelino et al. 2002; Goldman 2005). In fact, photometric variability has been detected in LP 944-20 at optical wavelengths (Tinney \& Tolley 1999). On the other hand, near-infrared monitoring of about 20 late $\mathrm{M}$ and $\mathrm{L}$ dwarfs failed to detect any variability (Bailer-Jones \& Lamm 2003; Koen et al. 2005).

LP 944-20 and other ultracool dwarfs dramatically violate the X-ray/radio-emission relation for normal stars, as its quiescent radio emission is $4-5$ orders of magnitude brighter than expected from its X-ray emission (Berger et al. 2005). The radio emission observed in some ultracool dwarfs implies a large magnetic field strength (over a hundred gauss averaged over the whole surface). Thus, while it is now clear that at least some old BDs have strong magnetic fields, the properties of the resulting coronae are strikingly different from those of stars. Possibly, not only the coronae differ from those of stars but also the structure of the magnetic field itself. As shown by several authors (Chabrier \& Kuker 2006; Dobler et al. 2006), fields of fully convective objects (like BDs) are expected not to be concentrated in small spots but to be distributed on a global scale. In addition, due to the low temperature of old $\mathrm{BDs}$, the degree of ionization in the atmosphere is very low, leading to a very low degree of the coupling between the magnetic field and the atmosphere. The coupling between the gas and the magnetic field is usually described in terms of the magnetic Reynolds number $\operatorname{Re}_{m}=l v / \eta$ (where $l$ is the length scale, $v$ is the velocity scale, and $\eta$ is the magnetic diffusivity; Priest 1982). While for the Sun $\mathrm{Re}_{m}$ is of the order of unity, for ultracool dwarfs $\operatorname{Re}_{m}$ could be as low as $10^{-15}$ to $10^{-20}$ (Meyer \& Meyer-Hofmeister 1999; Mohanty et al. 2002). If this is true, BDs with strong magnetic field should not have spots, and thus the gas flow in the atmosphere should not be effected by the magnetic field.

We propose here that the RV variability detected with UVES may be due to weather effects, i.e., variability of cloud coverage and/or flows, streams, or long-lived nonmagnetic spots in the upper photosphere of this object. The near-infrared observations probe deeper in the photosphere, and hence they are less affected by those motions or structures. The small amplitudes of the photometric and spectroscopic variability reported by Tinney \& Tolley (1999) and Guenther \& Wuchterl (2003), respectively, indicate that the surface features do not have a large contrast with the photosphere, and hence they are not like starspots.

Independently from a deep physical understanding of the cause of the RV variations in the optical, we can safely conclude on pure empirical grounds that future RV-based planet searches in late $\mathrm{M}$ stars and BDs will be better conducted at near-infrared wavelengths than in the optical because the targets are brighter and because the measurements are less affected by photospheric noise. This finding encourages the use of a new generation of high-resolution near-infrared spectrographs (Kaufl et al. 2004; Martín et al. 2005) for surveying the planetary population of very low mass stars and BDs.

Financial support was provided by the Michelson Science Center, NSF research grant AST 02-05862, and Spanish MEC grants AYA2003-05355 and AYA2005-06453. This Letter is partly based on observations obtained at the European Southern Observatory at Cerro Paranal, Chile, in programs 67.C-0160(A), 68.C-0063(A), and 072.C-0110(B). We thank the Keck and VLT observatories' staffs for their support. The W. M. Keck Observatory is operated as a scientific partnership between the California Institute of Technology, the University of California, and NASA. The Observatory was made possible by the generous financial support of the W. M. Keck Foundation. The authors extend special thanks to those of Hawaiian ancestry on whose sacred mountain we are privileged to be guests.

\section{REFERENCES}

Apai, D., Pascucci, I., Bouwman, J., Natta, A., Henning, Th., \& Dullemond, C. 2005 , Science, 310,834

Bailer-Jones, C. A. L., \& Lamm, M. 2003, MNRAS, 339, 477

Bailer-Jones, C. A. L., \& Mundt, R. 2001, A\&A, 374, 1071

Balthasar, H., Thiele, U., \& Woehl, H. 1982, A\&A, 114, 357

Barrado y Navascués, D., \& Martín, E. L. 2003, AJ, 126, 2997

Béjar, V. J. S., et al. 2001, ApJ, 556, 830

Berger, E., et al. 2001, Nature, 410, 338

. 2005, ApJ, 627, 960
Caccin, B., Cavallini, F., Ceppatelli, G., Righini, A., \& Sambuco, A. M. 1985, A\&A, 149, 357

Chabrier, G. 2003, PASP, 115, 763

Chabrier, G., \& Kuker, M. 2006, A\&A, 446, 1027

Dobler, W., Stix, M., \& Brandenburg, A. 2006, ApJ, 638, 336

Gelino, C. R., Marley, M. S., Holtzman, J. A., Ackerman, A. S., \& Lodders, K. 2002, ApJ, 577, 433

Goldman, B. 2005, Astron. Nachr., 326, 1059

Guenther, E. W., \& Wuchterl, G. 2003, A\&A, 401, 677 
Kaufl, H.-U., et al. 2004, Proc. SPIE, 5492, 1218

Klein, R., Apai, D., Pascucci, I., Henning, Th., \& Waters, L. B. F. M. 2003, ApJ, 593, L57

Koen, C., Tanabé, T., Tamura, M., \& Kusakabe, N. 2005, MNRAS, 362, 727

Kumar, S. S. 1963, ApJ, 137, 1121

Luhman, K., Adame, L., D’Alesio, P., Calvet, N., Hartmann, L., Megeath, S. T., \& Fazio, G. G. 2005, ApJ, 635, L93

Luyten, W. J., \& Kowal, C. T. 1975, A Catalogue of Stars with Proper Motions Exceeding 0.5 Annually (Minneapolis: Univ. Minnesota Press)

Magazzù, A., Martín, E. L., \& Rebolo, R. 1993, ApJ, 404, L17

Martín, E. L., \& Bouy, H. 2002, NewA, 7, 595

Martín, E. L., Delfosse, X., Basri, G., Goldman, B., Forveille, T., \& Zapatero Osorio, M. R. 1999, AJ, 118, 2466

Martín, E. L., Guenther, E., Barrado y Navascués, D., Esparza, P., Manescau, A., \& Laux, U. 2005, Astron. Nachr., 326, 1015

Martín, E. L., Rebolo, R., \& Zapatero Osorio, M. R. 1996, ApJ, 469, 706
Martín, E. L., Zapatero Osorio, M. R., \& Lehto, H. J. 2001, ApJ, 557, 822

Meyer, F., \& Meyer-Hofmeister, E. 1999, A\&A, 341, L23

Mohanty, S., \& Basri, G. 2003, ApJ, 583, 451

Mohanty, S., Basri, G., Shu, F., Allard, F., \& Chabrier, G. 2002, ApJ, 571, 469

Priest, E. 1982, Observatory, 102, 118

Ribas, I. 2003, A\&A, 400, 297

Roberts, D. H., Lehar, J., \& Dreher, J. W. 1987, AJ, 93, 968

Rutledge, R. E., Basri, G., Martín, E. L., \& Bildsten, L. 2000, ApJ, 538, L141

Scargle, J. D. 1982, ApJ, 263, 835

Smith, M. A. 1982, ApJ, 253, 727

Tinney, C. G. 1998, MNRAS, 296, L42

Tinney, C. G., \& Tolley, A. J. 1999, MNRAS, 304, 119

Zapatero Osorio, M. R., Martín, E. L., Bouy, H., Tata, R., Deshpande, R., \& Wainscoat, R. 2006, ApJ, submitted (astro-ph 0603194) 